POST-SHOCK SPIKES: A NEW FEATURE OF PROTON AND ALPHA

ENHANCEMENTS ASSOCIATED WITH AN INTERPLANETARY SHOCK WAVE

G. Gloeckler and F.M. Ipavich

University of Maryland, College Park, Mariyland 20742

and

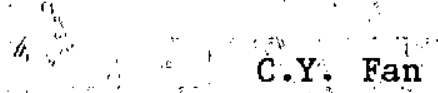

University of Arizona, Tucson, Arizona $85721^{\text {m }}$

, and

D. Hovestadt

Max Planck Institut, 8046 Garching, Germany

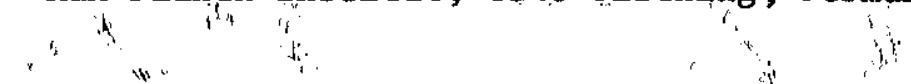

Technical Report 非4-115

June 1974
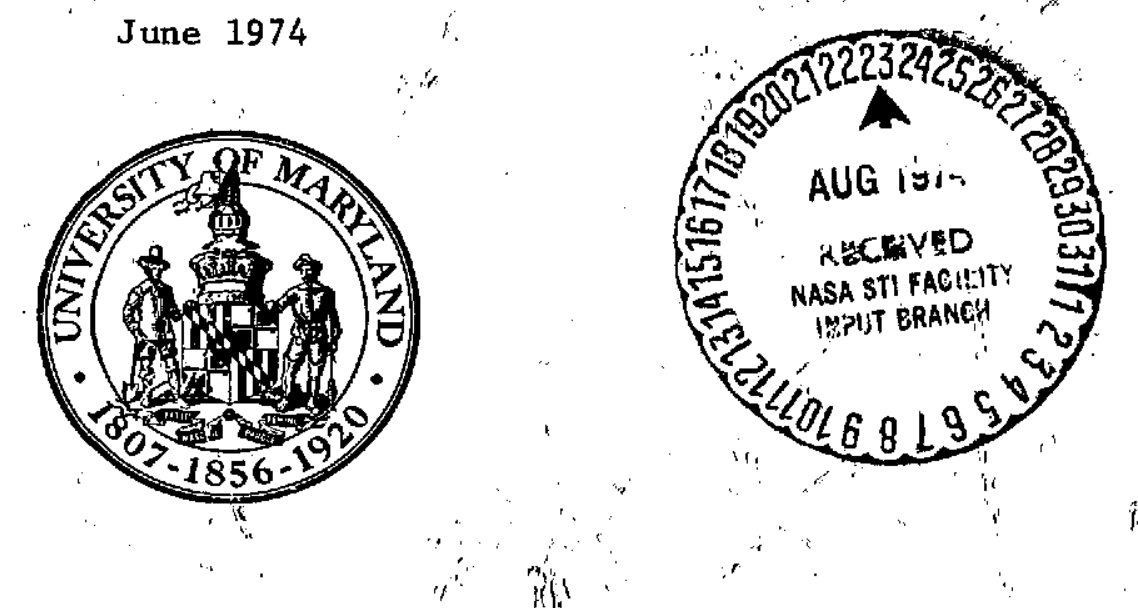

UNIVERSTTY OF MARYLAND

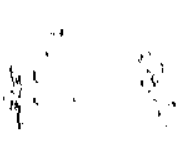

DEPARTMENT OF PHYSICS AND ASTRONOMY COLLEGE PARK, MARYLAND

Space Physics Group

(NASA-CR-139388) POST-SHOCK SPIKES: A NET FEATURE OF PROTON AND ALPEA

ENHANCEMENTS ASSOCIATED HITH AN

INTERPLANETARY SHOCK WAVE (MaIYland Univ.)

17. $\mathrm{p}$ HC $\$ 4.00$ 
This is a preprint of research carried out at the University of Maryland. In order to promote the active exchange of research results, individuals and groups at your institution are encouraged to send their preprints to

\section{PREPRINT LIBRARY}

\section{DEPARTMENT OF PHYSICS AND ASTRONOMY \\ UNIVERSITY OF MARYLAND \\ COLLEGE PARK, MARYLAND}

20742

U.S.A. 


\title{
POST-SHOCK SPIKES: A NEW FEATURE OF PROTON AND ALPHA \\ ENHANCEMENTS ASSOCIATED WITH AN INTERPLANETARY SHOCK WAVE*
}

\author{
G. Gloeckler and F.M. Ipavich \\ University of Maryland, College Park, Maryland 20742 \\ and \\ C.Y. Fan \\ University of Arizona, Tucson, Arizona 85721 \\ and \\ D. Hovestadt \\ Max Planck Institut, 8046 Garching, Germany \\ Technical Report \#74-115 \\ June 1974
}

* Published in Geophysica1 Research Letters, 1, 65, 1974. 
POST-SHOCK SPIKES: A NEW. FEATURE OF PROTON AND ALPHA ENHANCEMENTS ASSOCTATED WITH AN INTERPLANETARY SHOCK WAVE

G. Gloeckler and F.M. Ipavich

University of Maryland, College Park, Maryland 20742

and

C.Y. Fan

University of Arizona, 'Tucson, Arizona 85721

and

D. Hovestadt

Max Planck Institut, 8046 Garching, Germany

ABSTRACT

Abrupt and prolonged enhancements in the intensities of 100 to 22000 $\mathrm{keV} /$ nucleon protons and alphà particles observed in interplanetary space are interpreted as particle populations confined between an interplanetary shock front ard a magnetic field discontinuity. Prominent intensity "spikes" obsêrved only bèlow $\sim 400 \mathrm{keV}$ per charge for both protons and alpha particles several hours behind the shock front suggest that some fraction of the confined particles is accelerated by an energy per charge dependent process. 
Sudden increases in the intensity of low energy, interplanetary particles are frequently associated with the passage of flare generated shock waves, corotating standing shocks and magnetic field tangential discontinuities. Although these events vary considerably in their signature, two general types are recognized: (a) Energetic Storm Particle (ESP) events, having time scales on the order of hours and normally observed in association with an interplanetary shock front or tangential discontinuity in the interplanetary magnetic field (Bryant, et a1., 1962; Lanzerotti, 1969; Kahler, 1960; McCracken and Rao, 1970; Kunstmann and Wibberenz, 1973), and (b) "Shock-Spike" events with time scales of 5 to 30 minutes and typically occuring within 5 to 10 minutes of a shock front passage (Axford and Reid, 1963; Armstrong, et al., 1970; Ogilvie and Arens, 1971; Singer and Montgomery, 1971). Simultaneous measurements of protons and alpha particles at equal velocities, equal rigidities and equal energy per charge as a function of time have been recognized to be of fundamental importance in determining the probable causes for these enhancements (Lanzerotti and Robbins, 1969; Scholer et al., 1972).

In this letter we describe the salient characteristics of temporal variations of $\gtrsim 100 \mathrm{keV} /$ nucleon protons and alpha particles during a ten hour period associated with the passage of an interplanetary shock wave. In addition to extending measurements to energies considerably below previous observations for ESP and Shock-Spike events, we report the discovery of a new low energy feature which we will refer to as "Post-Shock Spikes". The typical width of these spikes is several minutes but they 
occur several hours after the passage of the shock front. We present evidence that the mechanisms responsible for these post-shock spikes depends on the energy per charge of the particles.

Our measurements were made in interplanetary space from October 29 to November 2, 1972 using the University of Maryland Electrostatic Deflection Spectrometer on board the IMP 7 (Explorer 47) spacecraft (Fan et a1., 1971). This instrument identifies low energy protons, alphas and heavier particles by simultaneously measuring the kinetic energy and amount of deflection of collimated incoming particles in a known electrostatic field. Table 1 lists the instrument rate characteristics relevant to the present discussion.

The ten minute averaged counting rate of $125-160 \mathrm{keV}$ protons is shown in Fig. 1 for the October 29 to November 2, 1972 time period. The slow Increase in intensity on days 304 and 305 is most likely due to the arrival at earth of low energy particles generated by the $2 \mathrm{~N}$ flare of the previous day. Of interest here is the shaded portion of the intensity vs. time profile. Coincident with the storm sudden conmencement (SC) we observe a "shock spike". The enhancement continues for about 8.5 hours ending at the time of the main phase (MP) of the geomagnetic storm. The most outstanding feature of the event is the set of three large but short lived intensity increases centered around 2000 hours on day 305, approximately 3 hours after the passage of the shock front. These are the "post-shock spikes".

In order to investigate the origin of these increases we plot in Fig. 2 the ten minute averaged differential intensity of 125 to 160,380 to 650 and 1150 to $1870 \mathrm{keV}$ protons as well as 95 to 135 and 310 to $465 \mathrm{keV} /$ nucleon alpha particles during the 12 hour period of the enhancement using a common time base. AlI five rate channels reach a maximum 
at time $T_{1}$ and decrease abruptly at $T_{2}$. The absence of velocity dispersion leads us to interpret the prolonged enhancement as a spatial feature moving past the satellite. The particle enhancement, especially prominent for the lowest energy protons, argues for confinement of particles between two well defined "barriers" in the interplanetary magnetic field. Indeed, examination of magnetic field data (Ness and Lepping, private communication, 1974) reveals the existence of an interplanetary shock at $\mathrm{T}_{1}$. At $\mathrm{T}_{2}$ a prominent magnetic field discontinuity was observed with the field component normal to the ecliptic plane changing from northward to southward, and the component in the ecliptic plane rotating by $180^{\circ}$ within two minutes. The fleld magnitude also changed marked1y, increasing from 16 to $23 \gamma$. The abrupt decrease in all channels at the time of the field discontinuity, $\mathrm{T}_{2}$, is interpreted to be caused by the passage of the satellite from the region populated by particles in the enhancement to one containing prlmarily solar flare particles. (See also Fig. 1.)

The large increases at $\mathrm{T}_{1}$ have time profiles not unlike the shockspike events described in the Iiterature (Og1lvie and Arens, 1971; Singer and Montgomery, 1971). Examining the proton and alpha rate profiles around $\mathrm{T}_{1}$ we notice the similarity between the $\mathrm{P} 2$ and $\mathrm{A} 1$ as well as the $\mathrm{P} 3$ and $\mathrm{A} 2$ channels, which have, respectively, identical rigidity responses (see Table 1). Even more revealing is the time behavior of the $\mathrm{p} / \alpha$ ratio computed in Fig. 3c, where we find the "equal rigidity" ratio at $30 \mathrm{MV}$ remains remarkably constant across the shock front at $\mathrm{T}_{l}$. We conclude, therefore, that below $60 \mathrm{MV}$ the process responsible for the shock spike is rigidity dependent. On the other hand, observations of other events at higher 
energies made by Lanzerotti and Robbins (1969) and Scholer et al. (1972) show the $\mathrm{p} / \alpha$ ratio to be constant across the shock front only when evaluated at equal energy per charge.

An entirely new feature of these observations is the discovery of large spikes occuring several hours after shock front passage. These "postshock" spikes have the following characteristics:

(a) They occur some 2 to 3 hours after the passage of the shock front (Fig. 2) .

(b) They are seen only in the lowest energy proton and alpha channels; no post-shock spikes are seen above $2400 \mathrm{keV}$ per charge. (Fig. 2)

(c) Across these post-shock spikes the $\mathrm{p} / \alpha$ ratio remains constant only when evaluated at equal energy per charge (Fig $3 b$ ); the ratio is about 20 at $230 \mathrm{kV}$.

(d) The width of the post-shock spikes is some 2 to 7 minutes corresponding to scale lengths of several times $10^{10^{\circ}} \mathrm{cm}$. The spike at $\mathrm{S}_{3}$ of Fig. 2 consists of four narrower spikes having nearly identical structures in both the protons and alpha channels.

(e) During the spikes the particle anisotropy increases dramatically; the streaming is generally unidirectional, field aligned and directed away from the shock front (Ipavich, 1974). To account for the persistent enhancement between the shock front at $\mathrm{T}_{1}$ and the field discontinuity at $\mathrm{T}_{2}$ we consider two processes. In 
the first, particles are accelerated (Fisk, 1971) or swept up (Palmer, 1972) by the moving shock front with some fraction leaking into the region behind the shock front. We note that such a snow plow mechanism would pile up particles ahead of the shock front, as observed (see Fig. 2), and is expected to depend on the particle rigidity (Fisk, 1971), consistent with our results for the shock-spike at $\mathrm{T}_{1}$ (Fig. 3c). Furthermore, the general slow decrease of the particle intensities from $T_{1}$ to $T_{2}$ (see Fig. 2) and the observed flow of all particles directed away from the shock front in the frame of the solar wind support our conclusion that most of the greater than about $30 \mathrm{MV}$ particles are leaking in across the shock front. This mechamism alone is not sufficient, however, to explain our observations for 125-160 keV protons (channel PI in Fig. 2), where the intensity after is considerably larger than before the shock front passage. Nor can it account for the presence of the post-shock spikes. We therefore suggest that, in addition to the "leaky-snow plow" mechanism, a significant fraction of the less than $230 \mathrm{MV}$ particles observed behind the shock front as well as essentially all of the particles in the post-shock spikes are locally accelerated by an energy per charge dependent mechanism. One possible mechanism is discussed in a future paper (Levy et al., 1974), where it is suggested that the acceleration is associated with active neutral sheets at or behind the shock front, and that the narrow post-shock spikes are observed whenever the magnetic field lines connect the satellite directly to these turbulent regions. Whether the source of the accelerated particles is the high energy tail of the solar wind distribution or solar flare particles cannot be decided from the present data, although we note with interest the similarity of our observed $p / \alpha$ ratio at equal energy 
per charge ( 20$)$ to that typically found in the quiet solar wind (Hundhausen, 1972). In future work it wil1 be important to examine in detail the composition of particles in the enhancement to decide on their origin.

The authors are grateful to J. Cain, J. Gigante, R. Lundgren, M. Pairel, and R. Sciambi for their contribution to the design, fabrication and testing of the IMP 7 experiment. We thank J: Dalton for the development of the computer programs used in data reduction and analysis. We have benefited from discussions with Drs. E.H. Levy, L.A. Fisk, L.F. Burlaga and J.J. O'Gallagher. We also thank Drs. N. Ness and R. Lepping for the use of their magnetic field data. This work was supported in part by the National Aeronautics and Space Administration under contract NAS5-11063 and grant NGR 21-002-224. 


\section{REFERENCES}

Armstrong, T.P., S.M. Krimigis, and K.W. Behannon, Proton fluxes at 300 $\mathrm{keV}$ associated with propagating interplanetary shock waves, J. Geophys. Res. , 75, 5980, 1970 .

Axford, W.I., and G.C. Reid, Increases in intensity of solar cosmic rays before sudden commencements of geomagnetic storms, J.Geophys. Res., 68, $1793,1963$.

Bryant, D.A., T.L. Cline, U.D. Desai, and F.B. McDonald, Explorer 12 observations of solar cosmic rays and energetic storm particles after the solar flare of September 28, 1961, J. Geophys. Res., 67, 4983, 1962.

Fan, C.Y., G. Gloeckler, and E. Tums, An electrostatic deflection vs. energy instrument for measuring interplanetary particles in the range 0.1 to $\sim 3 \mathrm{MeV} / \mathrm{charge,} \mathrm{Proc.} \mathrm{Inter.} \mathrm{Conf.} \mathrm{On} \mathrm{Cosmic} \mathrm{Rays,} \mathrm{Hobart,} 4$, $1602,1971$.

Fisk, L.A., Increases in the low-energy cosmic ray intensity at the front of propagating interplanetary shock waves, J. Geophys. Res., 76, 1662, 1971. Hundhausen, A.J., Coronal Expansion and Solar Wind, p. 98, Springer-Verlag, New York, 1972.

Ipavich, F.M., The Compton-Getting effect for low energy particles, submitted to Geophys. Res. Lett. , 1974.

Kahler, S.W., A comparison of energetic storm protons to halo protons, Solar Phys., 8, 166, 1969.

Kunstmann, J., and G. Wibberenz, The connection of energetic storm particle events to interplanetary magnetic field discontinuities, Proc. Inter. Conf. on Cosmic Rays, Denver, 2, 1638, 1973. 
Lanzerotti, L.J., Low-energy solar protons and alphas as probes of the interplanetary medium: the May 28, 1967, solar event, J. Geophys. Res. , 74, $2851,1969$.

Lanzerotti, L.J., and M.F. Robbins, Solar flare alpha to proton ratio changes following interplanetary disturbances, Solar Phys., 10, 212, 1969.

Levy, E.H., F.M. Ipavich, and G. Gloeckler, Possible acceleration of charged particles through the reconnection of magnetic fields in interplanetary space, to be pub1ished, 1974.

McCracken, K.G., and V.R. Rao, Solar cosmic ray phenomena, Space Sci. Rev. $11,155,1970$

Ogilvie, K.W., and J.F. Arens, Acceleration of protons by interplanetary shocks, I. Geophys. Res., 76, 13, 1971.

Palmer, I.D., Shock wave effects in solar cosmic ray events, Solar Phys., 27, 466,1972 .

Scholer, M., D. Hovestadt, and B. Hausler, Change of solar flare proton to alpha ratios during an energetic storm particle event, Solar Phys., 24, 475,1972 .

Singer, S., and M.D. Montgomery, Detailed directional and temporal properties of solar energetic particles associated with propagating interplanetary shock waves, J. Geophys. Res., 76, 6628, 1971. 
TABLE 1

CHARACTERISTICS OF ELECTROSTATIC DEFLECTION SPECTROMETER RATE CHANNELS

\begin{tabular}{|c|c|c|c|c|c|c|}
\hline \multirow{2}{*}{ Detector } & \multirow{2}{*}{$\begin{array}{c}\text { Geometrical } \\
\text { Factor } \\
\left(\mathrm{cm}^{2} \mathrm{sr}\right)\end{array}$} & \multirow{2}{*}{$\begin{array}{l}\text { Particle } \\
\operatorname{type}^{(a)}\end{array}$} & \multirow{2}{*}{$\begin{array}{l}\text { Charge } \\
\text { Threshold }\end{array}$} & \multicolumn{3}{|c|}{ Response ${ }^{(\mathrm{b})}$} \\
\hline & & & & $\begin{array}{l}\text { energy/charge } \\
\text { (keV/charge) }\end{array}$ & $\begin{array}{l}\text { energy/nucleon } \\
(\mathrm{keV} / \mathrm{nuc})\end{array}$ & $\begin{array}{l}\text { rigidity } \\
\text { (MV) }\end{array}$ \\
\hline P1 & 0.02 & protons & $Q \geq 1$ & $125-160$ & $125-160$ & $15.3-17.3$ \\
\hline $\mathrm{P} 2^{(\mathrm{c})}$ & 0.03 & protons & $Q \geq 1$ & $380-650$ & $380-650$ & $26.7-34.9$ \\
\hline $\mathrm{P} 3^{(\mathrm{c})}$ & 0.02 & protons & $Q \geq 1$ & $1150-1870$ & $1150-1870$ & $46.5-59.3$ \\
\hline $\mathrm{A} 1^{(c)}$ & 0.03 & alphas & $Q \geq 2$ & $190-270$ & $95-135$ & $26.7-31.8$ \\
\hline $\mathrm{A} 2^{(\mathrm{c})}$ & 0.02 & alphas & $Q \geq 2$ & $620-930$ & $310-465$ & $48.2-59.1$ \\
\hline
\end{tabular}

(a) Most common.

(b) Assuming fully stripped nuclei.

(c) Rate is sectored into four $90^{\circ}$ segments for anisotropy measurements in the ecliptic plane. 


\section{FIGURE CAPTIONS}

Figure 1. Counting rate for $125-160 \mathrm{keV}$ protons during days 303-307, 1972. The relevant optical flare data are indicated, with the arrows representing times of maximum phase. Associated X-ray bursts are indicated by " $X$ ", each arrow representing the time of maximum 1-8 $\bar{\AA}$ flux. SC, MP, and FD mark, respectively, the times of sudden commencement and main phase of a geomagnetic storm, and of a Forbush decrease observed by neutron monitors. The encircled numbers represent the daily $A_{p}$ index. Geomagnetic and solar events times are taken from the Solar-Geophysical Data Bulletins, nos. $340,341,344,345$. The insert shows that the satellite was outside the earth's bow shock during times when the rate was above background.

Figure 2. Ten minute averaged differential intensity time profiles for the three proton and two alpha particle channels during passage of the shock wave, corresponding to the shaded portion of Fig. 1. Three types of particle increases are observed: (a) the shock spike at $\mathrm{T}_{1}$, (b) an ESP enhancement from $T_{1}$ to $T_{2}$, and (c) a set of post-shock spikes at $S_{1}$, $\mathrm{s}_{2}, \mathrm{~s}_{3}$

Figure 3. Proton to alpha particle ratios during passage of the shock wave. (a) The ratio at equal velocity uses the P1 proton intensity and an interpolated alpha particle intensity using detectors Al and A2. (b) The ratio at equal energy per charge uses the Al alpha particle intensity and an interpolated proton intensity using detectors P1 and P2. (c) The ratio at equal rigidity uses detectors $\mathrm{P} 2$ and $\mathrm{Al}$. During post-shock spikes observed at times $s_{1}, s_{2}$ and $s_{3}$, the $p / \alpha$ ratio remains constant on $1 \mathrm{y}$ when evaluated at equal energy per charge. During the shock spike at $\mathrm{T}_{\mathrm{I}}$ only the "equal rigidity" ratio remains constant. Instrumental background in the alpha channels prevents extension of these plots beyond the $T_{2}$ boundary. 


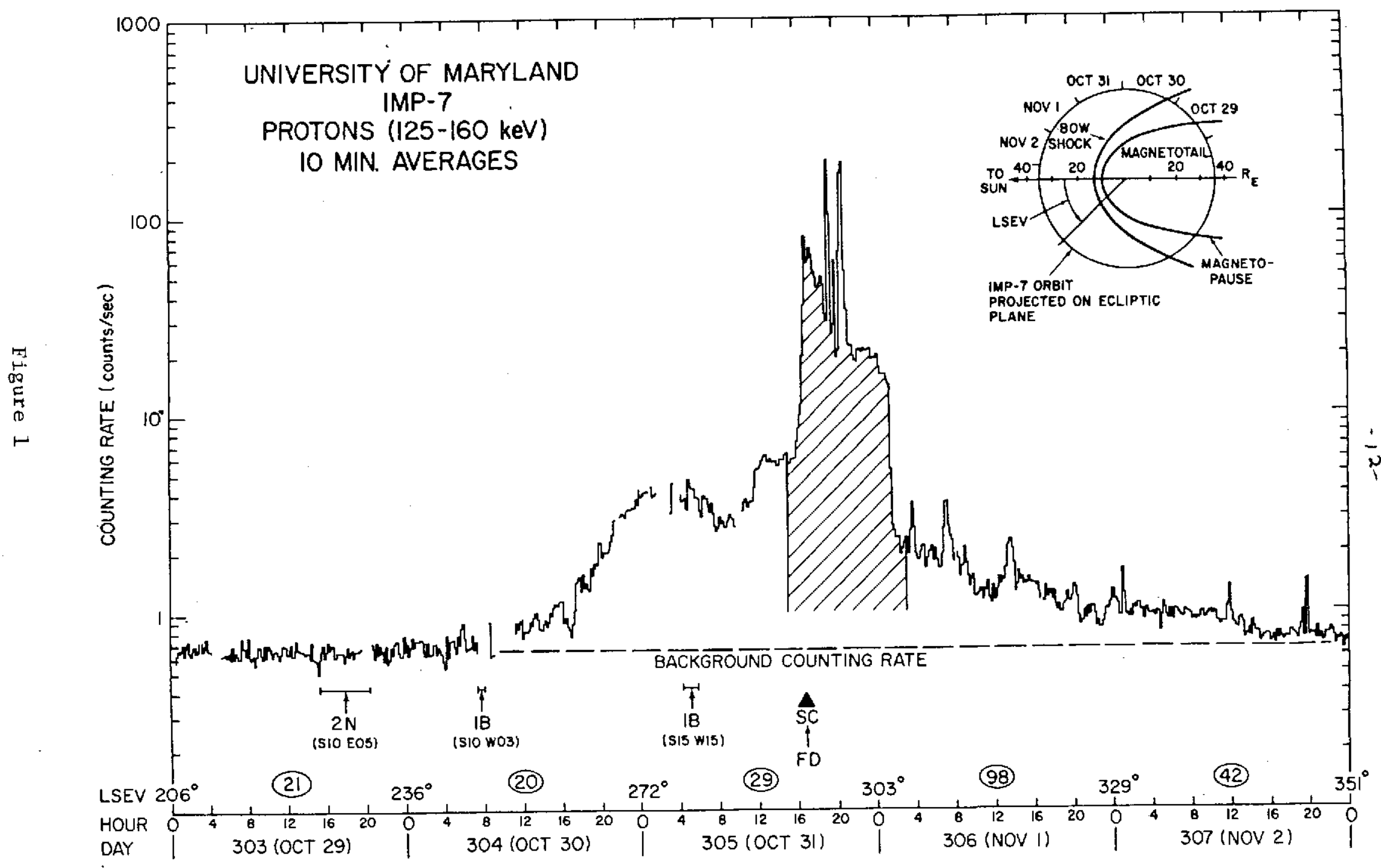




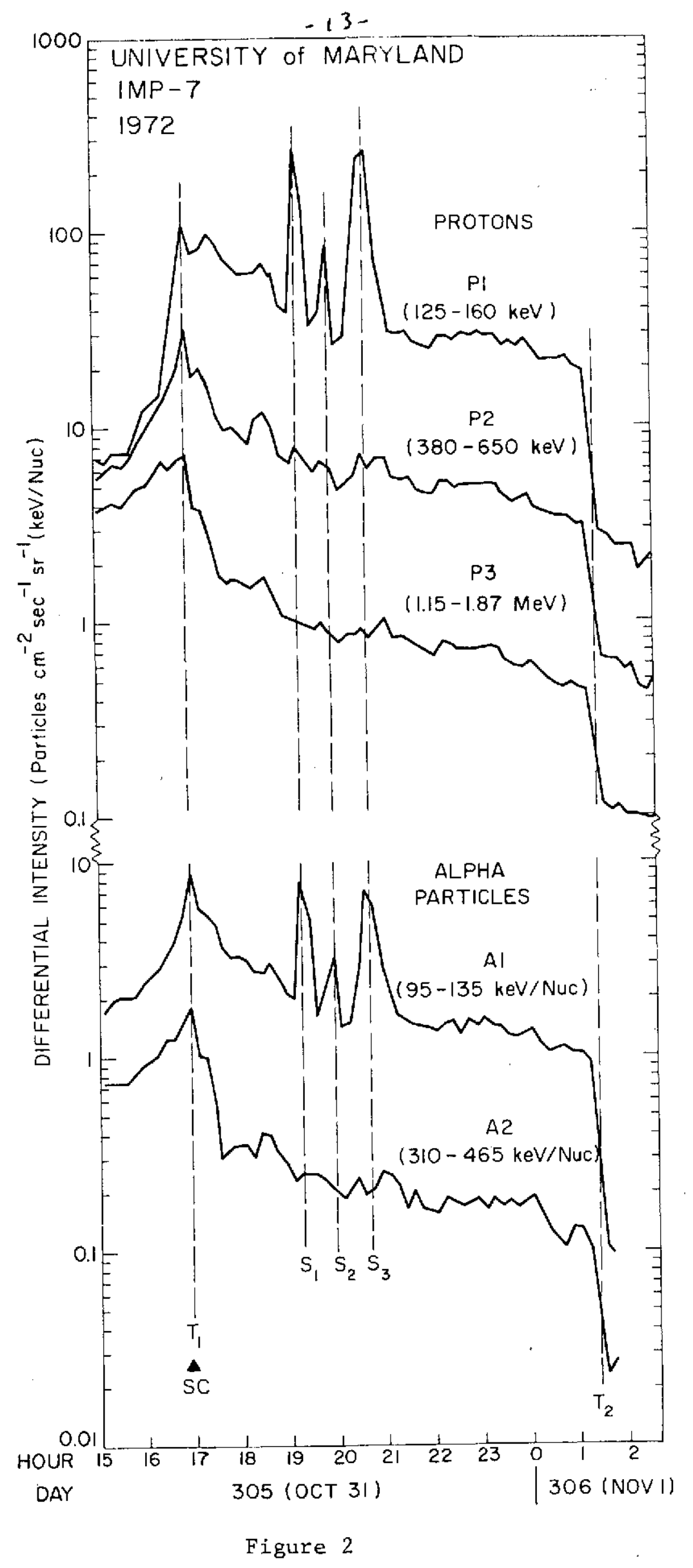




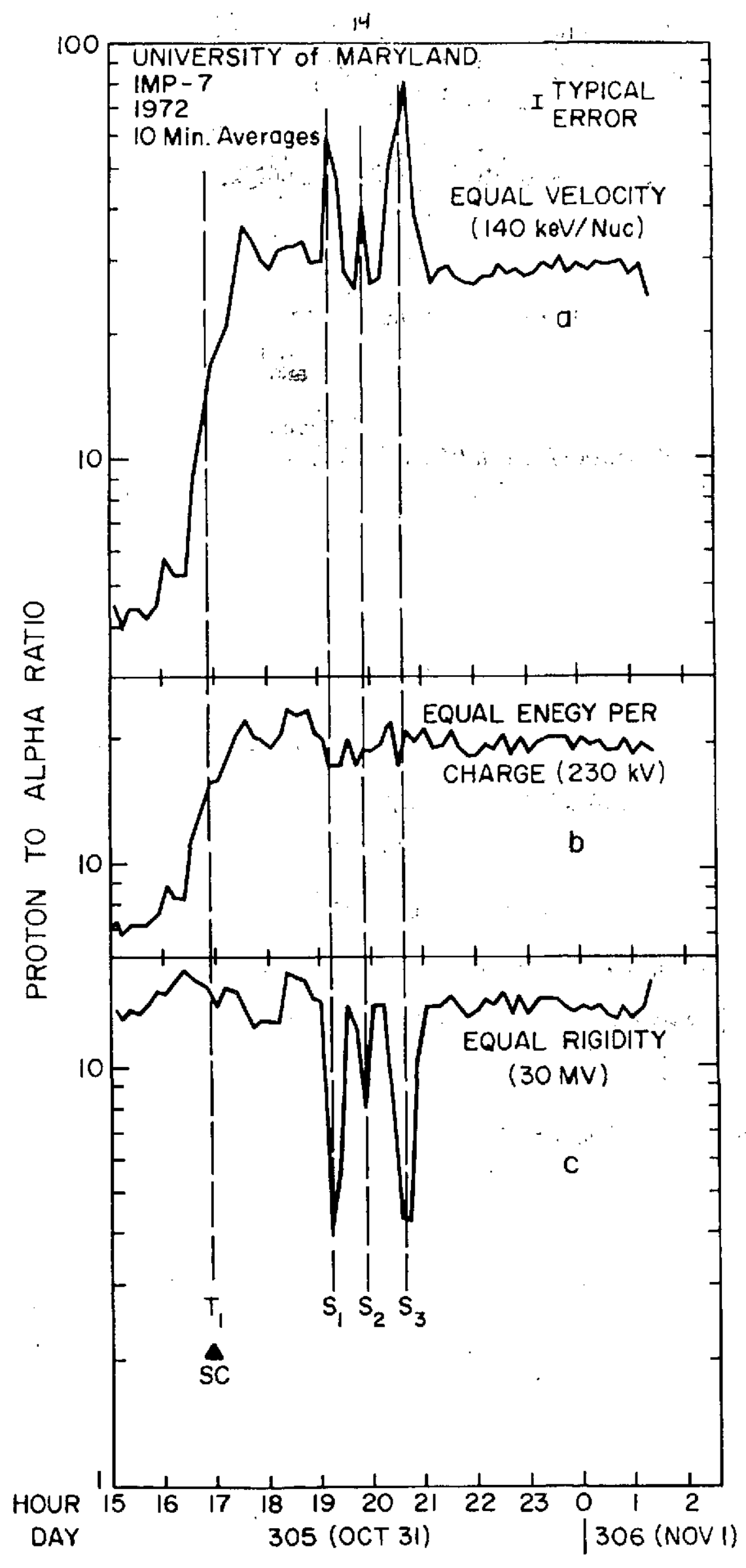

Figure 3 